\title{
UNA CARACTERIZACIÓN GENÉRICA DE LAS NOTICIAS DE LA PRENSA BRITÁNICA POR MEDIO DE LA COHESIÓN LÉXICA
}

\author{
ANA DÍAZ GALÁN \\ Universidad de La Laguna
}

\section{RESUMEN}

Este artículo persigue demostrar que las noticias de la prensa británica, indiferentemente a la clase de diario en que se publican, up-, mid- o down-market, presentan unas características genéricas comunes basadas en las relaciones semánticas que establecen sus elementos léxicos y en su organización textual que pueden ser probadas por medio de un análisis de la cohesión léxica como el diseñado por M. Hoey (1991). A la vista de los resultados estadísticos del análisis del corpus respecto a vínculos y conexiones léxicas, así como a su estructuración esquemática, se concluye que todos grupos de diario muestran las mismas características genéricas derivadas de su finalidad informativa, desmintiendo con ello disimilitudes debidas al formato o la clase social de los lectores.

Palabras clave: género, cohesión, cohesión léxica, noticias de prensa

\begin{abstract}
This paper tries to show that news items, even if published in different types of journal (up-mid or down-market), can be generically characterized on the basis of their textual organization and of the semantic relations established by their lexical items. Contrary to the traditional view that presupposes divergences between broadsheets and tabloids, using M. Hoey's (1991) analysis of lexical cohesion as a tool we will prove statistically that news items present common characteristics derived from their basic generic purpose, namely, providing information.
\end{abstract}

Key words: genre, cohesion, lexical cohesion, news items, news discourse 


\section{INTRODUCCIÓN}

Tradicionalmente, la prensa británica ha sido dividida en dos grupos de diarios conocidos como "cultos" y "populares", cuyas disparidades estriban no sólo en unas llamativas diferencias físicas, sino, también, en la clase social de sus lectores. Teniendo en cuenta investigaciones anteriores (Henry 1983, 1987), Jucker (1992) añadió a esta clasificación un tercer tipo, el mid-market, que tendría unas características específicas, aunque, a la vez, similares a las de los otros grupos, de la que se derivaría su posición intermedia. Además de confirmar por medios sociolingüísticos la existencia de esta clase de diarios, el trabajo de este autor descubre, también, que las divergencias entre las publicaciones no son tan llamativas como la tradición sugiere. De esta forma, según Jucker, aunque cada grupo muestra ciertas peculiaridades, todos comparten rasgos comunes que parecen derivarse, más que de su tipo, del lenguaje de la prensa en general.

Siguiendo su ejemplo, la intención de este artículo es comparar noticias de los tres grupos de diarios para descubrir si los elementos léxicos que contribuyen a su cohesión difieren y en qué medida definen su organización textual, buscando con ello, al tiempo, la caracterización general de las noticias de prensa como género.

Como instrumento de análisis utilizaremos el estudio de la cohesión léxica propuesto por M. Hoey (1991) ya que, a diferencia de otros análisis de este tipo de cohesión, no sólo presenta una catalogación de recursos cohesivos, sino que demuestra la relación entre éstos y la organización global de los textos, una aplicación que, a nuestro entender, parece especialmente adecuada para la descripción de las noticias. Respecto al corpus analizado, y puesto que éste se compone de un número reducido de textos ${ }^{1}$, no pretendemos aquí presentar resultados estadísticamente significativos; por medio de su comparación intentamos solamente apuntar una tendencia y, sobre todo, investigar hasta qué punto las

\footnotetext{
${ }^{1}$ El corpus analizado se compone de treinta noticias. Puesto que, por evidentes razones de espacio, no es posible reproducirlas nos limitamos aquí a enumerar sus títulos y el diario donde se publicaron el 12 de marzo de 1992. Los textos se han codificado con una letra, $a, b$ o $c$, según el grupo al que pertenece el diario donde se publicaron, - up, mid- o down market, y, un número, de acuerdo con los temas en los que han sido divididos, de forma que, por ejemplo, los textos 1a, 1b y 1c corresponden a la misma noticia publicada en tres tipos de diario diferente:

1a: "West threatens to bomb Iraqi nuclear plants for defying UN" The Times 1b: "Countdown to war as UN runs out of patience" Daily Mail 1c: "The Saddam factor" Daily Star 2a: "Slap in the face for a Tango ad" Daily Telegraph 2b: "TV ad scrapped after school slapping craze" Daily Express 2c: "Tango-ing! Telly Chiefs slap ban on Mr. Orange" The Sun 3a: "McDonald's woman in hoax bomb call" The Independent 3b: "Burger wars girl in hoax bomb call" Today 3c: "Doing a bomb in burgers" Daily Star 4a: "MPs admonish bank over BCCI" The Times 4b: "MPs admonish bank over massive BCCI fraud" Daily Express 4c: "Major BCCI rap" Daily Mirror 5a: "Carey condemned as 'ayatollah' over gay book" The Guardian 5b: "Gays attack 'ayatollah' archbishop" Daily Express 5c: "Gay prayer book fury" Daily Star 6a: "Nurse faces new charges of attempted murder" Daily Telegraph 6b: "Nurse accused of hospital killings faces new charges" Daily Mail 6c: "Nurse on new charges" Daily Mirror 7a: "Country cottage was drugs center"" The Guardian 7b: "Cops turn dustmen to crack LSD gang" Today 7c: ,60m LSD factory at Lawless Cottage" Daily Mirror 8a: "Woman sinks pool opponents" The Guardian 8b: "Susan wins the right to cue up for pool room" Today 8c: "Cue ace Sue wins battle to turn pro" The Sun 9a: "TV rivals fire first salvoes in battle for election" The Guardian 9b: "Battle of TV Experts" Daily Express 9c: "Major ducks live TV head-to-head" Daily Mirror 10a: "Bush backing for "superb' Major" The Guardian 10b: "A pat on the back from Bush" Daily Express 10c: "Major is a superb PM says Bush" Daily Star.
} 
relaciones cohesivas léxicas podrían ser definitorias para el género de las noticias. Basándonos en la opinión de Biber, podemos justificar que este tipo de acercamiento microscópico puede ser adecuado para los fines que perseguimos:

Microscopic text analysis is necessary to pinpoint the exact communicative functions of individual features. It complements macroscopic analysis in two ways: (1) it identifies the potentially important linguistic features and genre distinctions to be included in macro-analysis, and (2) it provides detailed functional analyses, which enable interpretation of the textual dimension in functional terms (Biber 1988:62).

Antes de proceder a la comparación que, por tanto, constituye nuestro objetivo, en las siguientes secciones intentaremos caracterizar las noticias de prensa como género así como explicar en detalle el método e instrumentos de análisis empleados.

\section{LAS NOTICIAS DE LA PRENSA COMO GÉNERO}

Si, siguiendo a van Dijk (1990:17), llamamos noticia a cualquier documento periodístico que nos informe acerca de hechos recién acaecidos o que nos aporte nuevos conocimientos acerca de un tema vigente o de actualidad, en virtud de su función comunicativa reconocida, éstas podrían ser claramente catalogadas como género.

De acuerdo con Eggins, la funcionalidad, establecida culturalmente, es la característica definitoria de un género: "Wherever language is being used to achieve a culturally recognized and culturally established purpose, there we find genre" (1994:47). Esta autora, igual que otros dentro la Gramática Sistémico Funcional (Halliday 1994; Hasan 1989; Martin 1992), entiende el género como una de las maneras de dar cuenta del contexto cultural general, sin olvidar por ello que el género se codifica o se realiza por medio del lenguaje con la mediación del registro, elemento que, a su vez, se ocupa del contexto situacional a través de las variables campo, modo y tenor.

Sin embargo, en el establecimiento de lo que constituye un género, además de la premisa básica de la función, se pueden incluir, tal como afirma Swales, otros factores: "In addition to purpose, exemplars of a genre exhibit various patterns of similarity in terms of structure, style, content and intended audience" (1990:58). Para Paltridge, en cambio, el género puede ser abordado como un evento comunicativo que conlleva ciertas implicaciones:

Our perception of the concept of genre is an instance of a particular communicative event [...]. The interactional characteristics of a genre may include specific author/s and audiences of the text, a particular channel of communication at certain level of abstraction, a particular "topic" and a social stock of knowledge. The communicative event, furthermore, occurs in a particular social and cultural setting and has a particular communicative function. Co-occurring with this interactional frame is also a cognitive frame which incorporates a certain conceptual scenario. This scenario may include certain roles, co-occurring patterns of textual organization and shared understandings, or protocols, of how the text may proceed. There may also be certain lexical items which are associated with the particular genre (1997:106). 
Las noticias de prensa escrita responderían a las características de género descritas anteriormente en tanto representan un evento comunicativo que presupone un autor concreto, una audiencia preestablecida y bien estudiada por los diarios por razones económicas, un canal predeterminado, un tema específico y, además, una localización social y cultural propia que, en ocasiones, lleva implícita una ideología particular (van Dijk 1987; Fowler 1991). Factores estos que, en su conjunto, pueden influir también en lo que se conoce como el estilo de la prensa (Crystal y David 1968; van Dijk 1990; Downing 1998).

Otra de las características definitorias del género, en este caso estrictamente linguiística, es la presencia de ciertos elementos léxicos. Para Hasan (1989) o Martin (1992), el léxico y las relaciones léxicas, entre ellas la cohesión, se integran en el campo, field, y contribuyen definitivamente a establecer el género. Aunque Paltridge disiente de los dos sistemicistas en algunos de los conceptos relativos al género, abunda en la misma idea, resaltando que el léxico tiene un papel relevante al respecto y enfatizando que las conexiones textuales que conocemos como cohesión léxica, es decir la relación semántica o taxonómica entre los vocablos de un texto, son, también, un indicativo de género, una posibilidad no contemplada, hasta ahora, en las noticias: "The place of associated words within a framework for genre identification thus concerns not just the occurrence of lexical items across instances of particular genres, but also subject-specific words which are in a semantic or taxonomic relationship with each other" (Paltridge 1997:83).

Por último, una de las reglas comúnmente aceptadas para la identificación de un género es que los ejemplares del mismo deben poseer una estructura esquemática similar (Swales 1990). Las exigencias macroestructurales de organización, que pueden ser más o menos flexibles según los tipos de texto y los autores, también se cumplen en las noticias, si atendemos a la conocida estructuración que ha propuesto van Dijk (1977, 1988, 1990) y que abordaremos en el siguiente apartado.

\subsection{La organización esquemática de la noticia}

La descripción del discurso periodístico realizado por van Dijk (1977, 1988, 1990, etc.) se fundamenta en que las noticias poseen no sólo contenido sino, también, forma. Según esta visión formal, el texto informativo se ordena de acuerdo con un conjunto de reglas conocidas como news schemata, un esquema específico y definitorio del género de las noticias que existe previamente a su creación. Este esqueleto de la noticia, el que se insertaría el contenido, está organizado igual que una sintaxis, de manera que la presencia o no de ciertos elementos y su posición en la estructura no es aleatoria.

El news schemata se representa normalmente como un diagrama. En su parte superior hallaremos las categorías más importantes de la noticia: el resumen, compendio de los eventos principales similar al abstract, y el cuerpo propiamente dicho, donde se incluyen el episodio y los comentarios. Todos ellos comprenden distintas subcategorías y, así, el resumen incluye el titular/es, donde se condensa el tema principal de la noticia, y la entradilla, cuya función es repetir de forma más explícita la información proporcionada por el titular. Ya en el cuerpo de la noticia, el episodio abarca el evento principal, aquel que ha motivado la noticia, y, opcionalmente, uno o más eventos secundarios ordenados 
de acuerdo con su relevancia. Tras el evento principal suelen aparecer sus antecedentes, en forma de historia (información acerca de hechos pasados relacionados) o de circunstancias (a su vez, éstas pueden consistir en un contexto, es decir, información sociopolítica, o en hechos previos, información sobre eventos anteriores) y, las consecuencias o reacciones (verbales of factuales) al evento principal. Por último, los comentarios acerca de la información pueden proporcionarse como expectativas, es decir, posibilidades para el futuro, o como evaluaciones, opiniones personales del periodista o del diario.

Estas categorías no poseen el mismo rango, de forma que, en teoría, una noticia, para serlo, sólo requiere un titular y un evento principal, lo que convierte a estos elementos en los más importantes del esquema. También, de acuerdo con van Dijk (1990:93), el contexto y las reacciones verbales son estadísticamente significativos.

Las noticias se distinguen por poseer una disposición particular de la información motivada por su proceso de creación y por los valores que se le asocian (Bell 1991a:14855), como la claridad, la brevedad, la novedad o la relevancia (los news values de Galtung y Ruge 1973). El orden de aparición de las distintas informaciones de una noticia está, por tanto, condicionado por su relevancia, de manera que es posible comenzar a tratar un asunto, abandonarlo y retomarlo más tarde. Puesto que los temas y las categorías de la noticia están relacionadas, también éstas últimas se pueden realizar en el texto de forma discontinua. En consecuencia, las noticias se organizan según los grados de generalidad o relevancia y la especificidad de los eventos (van Dijk 1983:87), pudiéndose decir, por tanto, que se ordenan jerárquica y cíclicamente y se realizan de forma recursiva.

\section{TIPOLOGÍA DE LOS DIARIOS}

La existencia de diferencias significativas entre las publicaciones diarias británicas, paradigma de las cuales son los diarios The Times y The Sun, es comúnmente conocida. El grupo representado por el primero se denomina prensa "culta" o de "calidad", publicaciones serias interesadas por temas políticos o económicos; mientras, el otro se ha llamado prensa "popular" por centrarse en los aspectos más escandalosos o personales de la noticia. Estas diferencias se subrayan, además, por el aspecto físico, al editarse la prensa "culta" en el formato broadsheet o sábana y la "popular" en el tabloid o tabloide. Sin embargo, ni esta subdivisión ni su denominación parecen ser las más adecuadas para dar cuenta de sus diferencias reales. Para clasificar los diarios deben considerarse factores objetivos que tengan en cuenta de forma consistente aspectos inherentes a la producción de los mismos, como su formato o el tratamiento de la información, así como otros socioeconómicos relativos al número y clase social de sus lectores. Basándose en ellos, es posible hacer una división de la prensa diaria británica en tres grupos, up-, mid-y down- market (Henry 1983, 1987): 


\begin{tabular}{|c|c|c|c|c|c|}
\hline \multirow{2}{*}{\multicolumn{2}{|c|}{$\begin{array}{c}\text { Sábana } \\
\text { Up-Market }\end{array}$}} & \multicolumn{4}{|c|}{ Tabloide } \\
\hline & & \multicolumn{2}{|c|}{ Mid-Market } & \multicolumn{2}{|c|}{ Down-Market } \\
\hline \multicolumn{2}{|c|}{ The Daily Telegraph (DT) } & Daily Express & $(D E)$ & Daily Mirror & $(D M i)$ \\
\hline Financial Times & $(F T)$ & Daily Mail & $(D M a)$ & Daily Star & $(D S)$ \\
\hline The Guardian & $(G)$ & Today & $(T o)$ & The Sun & $(S)$ \\
\hline The Independent & $(I)$ & & & & \\
\hline The Times & $(T)$ & & & & \\
\hline
\end{tabular}

Tabla 1: Clasificación socioeconómica de los diarios británicos

El formato no sólo sirve para establecer una división inicial entre los diarios, sino que, además, tiene repercusiones en su lenguaje. Ya Crystal y Davy (1969) hicieron notar que la disposición de los párrafos, en parte motivada por el tamaño de la página, hace que en la prensa tabloide se empleen con más frecuencia oraciones coordinadas que subordinadas. Más recientemente, se ha comprobado que este factor también influye en el terreno del análisis ideológico, bien por resaltar unos contenidos sobre otros, bien por la restricción en la información que impone un espacio reducido (van Dijk 1990; Kress y van Leeuwen 1998). En el aspecto puramente lingüístico, Jucker (1992) hace notar que existen diferencias generales en cuanto a la complejidad de los sintagmas nominales entre los broadsheet y los tabloides. Desde nuestro punto de vista, el formato de los diarios podría afectar a la cohesión léxica, en tanto los diarios de formato tabloide podrían contener un número inferior de conexiones léxicas.

Otros aspectos internos que sirven como diferenciadores de los diarios son las secciones en que suelen dividirse, sus temas, las clases de noticia que contienen y, sobre todo, el tratamiento de las mismas, que varía de forma proporcional a la popularidad del mismo, de manera que cuanto más "popular" sea el diario más personal y sensacionalista será este. Sin embargo, el elemento que contribuye más claramente a la distribución de los diarios en tres grupos es la clase social de sus lectores.

Por motivos económicos, cada tipo de diario se dirige a unos sectores específicos de la población; especialmente clara parece la asociación de las clases sociales más altas con los diarios up-market y la de las más bajas (clase trabajadora) con los down-market. El caso de los mid-market, como su nombre sugiere, es ciertamente intermedio, ya que ni todos los diarios muestran el mismo perfil de lectores, ni los lectores de clase media-baja, los que supuestamente deberían corresponderle, tienen preferencias claras por ningún tipo de diario (Díaz Galán 2004). Por último, la clase social de los lectores, es también importante por razones lingüísticas. La audiencia específica no sólo es uno de los rasgos definitorios del género, sino que en el caso de la prensa, influye en su estilo, por ejemplo, en términos de formalidad e informalidad (Bell 1991b; Jucker 1992). 


\section{MÉTODO: EL ANÁLISIS DE LA COHESIÓN LÉXICA DE M. HOEY}

El análisis contrastivo que nos proponemos llevar a cabo persigue demostrar que las noticias de prensa, indiferentemente a su clase de diario, presentan unas características genéricas comunes basadas en el tipo de relaciones semánticas que establecen sus elementos léxicos y en su organización textual, hechos que, en nuestra opinión, pueden ser probados por medio de un análisis de la cohesión léxica como el diseñado por M. Hoey (1991).

La cohesión léxica ha sido desde hace ya varias décadas (Halliday y Hasan 1976; Gutwinsky 1976) considerada imprescindible para la unidad del texto. Sin embargo, muchas de las aproximaciones a este concepto se basan meramente en la clasificación de los recursos cohesivos léxicos, descuidando la faceta textual que autores como Hasan (1989) han intentado resaltar. Basándose, además de en la investigación de la propia Hasan en otras sobre la relevancia del léxico (Winter 1979; Phillips 1989), Hoey ha demostrado la existencia de una interdependencia entre la cohesión y la organización global del texto.

Este autor defiende que los textos se organizan por medio de la reiteración de la información contenida en sus oraciones y que tal relación se materializa a través de lo que denomina conexiones léxicas. Estas son fundamentalmente repeticiones léxicas en diferentes variantes que fluctúan desde la iteración de un mismo elemento, hasta la elipsis o repetición cero², según se ve en la tabla 2 (Hoey 1991:51 y ss.):

\begin{tabular}{c|c}
\hline Tipos de Repetición & Ejemplos \\
\hline Simple lexical repetition & bear - bear \\
Complex lexical repetition & human (nombre) - human (adjetivo) \\
Simple paraphrase & produce - cause \\
Complex paraphrase & Author - writings \\
Substitution & author-he \\
Co-reference & Augustus - The Emperor \\
Ellipsis & author $-\varnothing$ \\
\hline
\end{tabular}

Tabla 2: Tipos de repetición en el modelo de M. Hoey

Puesto que su fin último es mostrar la organización textual, la identificación de estos tipos de repetición es sólo un primer paso y no el objetivo final del método. Una vez

\footnotetext{
${ }^{2}$ Todos los autores relevantes en la materia, desde Halliday y Hasan (1976) hasta el propio Hoey (1991), se han visto obligados a incluir relaciones gramaticales como la substitución, la co-referencia e, incluso, la elipsis dentro de la cohesión léxica. En el caso específico de Hoey, éstas se intentan restringir en la medida de lo posible, dando prioridad, en los casos donde existe elección, a las conexiones estrictamente léxicas.
} 
reconocidas las conexiones se ha de proceder a relacionar las oraciones en las que éstas aparecen. Para que la relación entre dos oraciones, próximas o no, sea textualmente significativa debe existir entre ellas un vínculo, es decir, un número de conexiones superior a tres. Las oraciones vinculadas se interconectan formando redes que descubren no sólo la organización temática del texto (dónde comienzan o finalizan los temas), sino, también, sus patrones textuales (por ejemplo, una oración que exprese causa estará conectada a otra/s que exprese/n consecuencia). Finalmente, siempre de acuerdo con Hoey, las oraciones que tienen más conexiones y vínculos son las más relevantes para el texto, considerándose centrales para el significado del mismo, mientras que las que contienen pocos o ninguno serían marginales. La prueba de su centralidad o marginalidad reside en el hecho de que las oraciones sin vínculos pueden eliminarse del texto sin causar cambios discernibles en su significado, mientras que uniendo las oraciones centrales entre ellas se obtienen subtextos (resúmenes o extractos con el mismo tema) coherentes.

\section{COMPARACIÓN DE LOS TIPOS DE REPETICIÓN LÉXICA}

Si, como sugiere Paltridge, las relaciones taxonómicas o semánticas entre los elementos léxicos son definitorias para el género, el resultado de aplicar el método resumido anteriormente al corpus avalaría la afirmación de que las noticias muestran rasgos de género relacionados con la cohesión léxica indiferentes a los grupos socioeconómicos de la prensa británica. Este hecho puede ser probado recurriendo a la comparación de la cantidad de vínculos y conexiones y al tipo de estas últimas. Como primer paso ofrecemos los datos generales respecto a palabras, elementos léxicos, conexiones y vínculos:

\begin{tabular}{lcccccccc}
\hline & \multicolumn{2}{c}{ up-market } & \multicolumn{2}{c}{ mid-market } & down-market & \multicolumn{2}{c}{ Total } \\
\hline & $\mathrm{n} 1$ & $\%$ & $\mathrm{~N} 1$ & $\%$ & $\mathrm{~N} 1$ & $\%$ & $\mathrm{n} 1$ & $\%$ \\
\hline Palabras & 2871 & 39,9 & 2732 & 38 & 1579 & 21,9 & 7182 & 100 \\
Els. léxicos & 1633 & 39,3 & 1590 & 38,3 & 922 & 22,2 & 4145 & 100 \\
Conexiones & 1452 & 39,9 & 1571 & 42,5 & 666 & 18 & 3689 & 100 \\
Vínculos a & 423 & 41,4 & 431 & 42,2 & 166 & 16,2 & 1020 & 100 \\
\hline
\end{tabular}

Tabla 3: $\mathrm{N}^{\mathrm{o}}$ y \% de palabras, elementos léxicos, conexiones y vínculos por grupos

Una aproximación inicial a ellos muestra, en todos los parámetros, una gran similitud entre las cifras totales y porcentajes de los diarios up- y mid-market, especialmente llamativa si tenemos en cuenta el hecho de que poseen un formato diferente. La extensión de los artículos, en contra de la opinión habitual, no parece estar motivada por el formato del periódico, hecho que se demuestra claramente al contrastar el número de palabras de cada 
uno de los grupos y que demostraría que el formato tabloide carece de influencia al respecto. Este mismo factor, junto a los resultados del resto de los parámetros, desmentiría la semejanza entre los dos tipos de prensa tabloide y, en cambio, la probaría entre la prensa de formato sábana y los diarios de mercado medio, al presentar ambos unas cifras casi idénticas. No significa esto, sin embargo, que los diarios de tipo down-market sean completamente diferentes al resto en sus relaciones léxicas. Lo único que se puede probar con certeza por medio de la tabla anterior es la menor extensión de este grupo de artículos, lo cual, implica, evidentemente, una posibilidad de aparición de conexiones y vínculos inferior al resto. La verdadera medida de la semejanza o desigualdad entre los grupos debe ser el cruce interno entre las variables. Los siguientes dos cuadros contienen los resultados de la combinación de los datos expuestos en la tabla previa:

\begin{tabular}{lccccccccc}
\hline & up-market & \multicolumn{2}{c}{ mid-market } & down-market & \multicolumn{2}{c}{ Media } \\
\hline & $\mathrm{n} 1$ & $\%$ & $\mathrm{n} 1$ & $\%$ & $\mathrm{n} 1$ & $\%$ & $\mathrm{n} 1$ & $\%$ \\
\hline $\begin{array}{l}\text { Palabras/ Els. Léxicos } \\
\text { Conexiones/ Els. }\end{array}$ & 1,7 & 56,8 & 1,7 & 58,1 & 1,7 & 58,3 & 1,7 & 57,7 \\
$\begin{array}{l}\text { léxicos } \\
\text { Conexiones/vínculos }\end{array}$ & 3,4 & 88,9 & 1 & 98,8 & 1,3 & 72,2 & 1,1 & 88,9 \\
Vínculos/ Els. léxicos & 3,8 & 25,1 & 3,6 & 27,4 & 4 & 24,9 & 3,6 & 27,6 \\
\hline
\end{tabular}

Tabla 4: Cruce de los resultados generales en todos los grupos

\begin{tabular}{|c|c|c|c|c|c|c|}
\hline & \multicolumn{6}{|c|}{ Variación } \\
\hline & \multicolumn{2}{|c|}{ up-/mid- } & \multicolumn{2}{|c|}{ up-/down- } & \multicolumn{2}{|c|}{ mid-/down- } \\
\hline & $\mathrm{n} 1$ & $\%$ & $\mathrm{n} 1$ & $\%$ & $\mathrm{n} 1$ & $\%$ \\
\hline Palabras/ Elementos léxicos & 0 & $-1,3$ & 0 & $-1,3$ & 0 & $-0,2$ \\
\hline Conexiones/Elementos léxicos & $+0,1$ & $-10,9$ & $+0,2$ & $+16,7$ & $+0,3$ & $+26,6$ \\
\hline Conexiones/vínculos & $-0,4$ & $+1,7$ & $+0,6$ & $+4,2$ & $+0,4$ & $+2,5$ \\
\hline Vínculos/ Elementos léxicos & $+0,2$ & $-1,2$ & $-1,7$ & $+7,9$ & $-1,9$ & $+9,1$ \\
\hline
\end{tabular}

Tabla 5: Cruce de los resultados. Variación entre grupos

La densidad léxica, es decir, la proporción entre palabras y elementos léxicos, nos muestra a priori la capacidad de cada tipo de diario de establecer conexiones. Examinando las cifras anteriores se puede comprobar que en todos los grupos ésta es idéntica en términos absolutos $(1,7)$ y porcentualmente muy similar, aunque, curiosamente, la de los diarios tipo sábana es ligeramente inferior a la de los diarios tabloides. De acuerdo con 
estos datos, más de la mitad de las palabras de los artículos analizados son elementos léxicos y, como media, el 88,9\% de ellos establecen conexiones. Esta cifra es incluso superior en los diarios mid-market, donde la ratio conexiones/elementos léxicos es de 1, o sea, una conexión por elemento léxico. El resultado en los diarios up-market es idéntico a la media y algo inferior en los down-market, a los que les correspondería una conexión cada 1,3 elementos léxicos. En la comparación entre la cantidad de conexiones y de vínculos destaca la prensa de formato sábana, aunque, las cifras son muy similares en todos los casos. Por último, la frecuencia de vínculos en relación con los elementos léxicos favorece, de nuevo, a la prensa de tipo medio y señala, por su menor frecuencia, a la de mercado bajo.

En resumen, podríamos decir que en dos de los parámetros, la relación palabras/elementos léxicos y conexiones/vínculos, los tres tipos de diarios están igualados, mientras que en los dos restantes, el contraste de los elementos léxicos con las conexiones y los vínculos, el grupo intermedio tiene los mejores porcentajes y el bajo los peores. De cualquier modo, ninguno de ellos es excesivamente llamativo. La ligera divergencia en los parámetros de la prensa down-market respecto a la cantidad de conexiones y vínculos en comparación con los elementos léxicos podría implicar que en este tipo de diarios las conexiones léxicas son más significativas. Nótese que la prensa down-market posee tantos vínculos, el elemento decisivo para la organización del texto, como cualquier otro tipo de diarios, aunque los establece con un número menor de conexiones y que, por tanto, éstas son más relevantes o decisivas para la organización del texto.

La comparación de los tipos de repetición utilizados en cada uno de los grupos de diarios, segundo parámetro de comparación, tampoco ha mostrado diferencias radicales:

\begin{tabular}{|c|c|c|c|c|c|c|c|c|}
\hline & \multicolumn{2}{|c|}{ up-market } & \multicolumn{2}{|c|}{ mid-market } & \multicolumn{2}{|c|}{ down-market } & \multicolumn{2}{|c|}{ todos } \\
\hline & $\mathrm{N} 1$ & $\%$ & $\mathrm{n} 1$ & $\%$ & $\mathrm{n} 1$ & $\%$ & $\mathrm{n} 1$ & $\%$ \\
\hline $\mathrm{Rs}$ & 716 & 49,3 & 785 & 49,9 & 255 & 38,2 & 1756 & 47,6 \\
\hline $\mathrm{Rc}$ & 217 & 14,9 & 180 & 11,4 & 64 & 9,6 & 461 & 12,4 \\
\hline Ps & 152 & 10,4 & 198 & 12,6 & 93 & 13,9 & 443 & 12 \\
\hline Psm & 55 & 3,8 & 77 & 4,9 & 30 & 4,5 & 162 & 4,4 \\
\hline Psp & 97 & 6,6 & 121 & 7,7 & 63 & 9,4 & 281 & 7,6 \\
\hline $\mathrm{Pc}$ & 95 & 6,5 & 113 & 7,1 & 46 & 6,9 & 254 & 6,9 \\
\hline Cor & 164 & 11,2 & 186 & 11,8 & 114 & 17,1 & 464 & 12,5 \\
\hline $\mathrm{S}$ & 96 & 6,6 & 108 & 6,8 & 92 & 13,8 & 296 & 8 \\
\hline E1 & 12 & 0,8 & 1 & 0,1 & 2 & 0,3 & 15 & 0,5 \\
\hline Total & 1452 & 100 & 1571 & 100 & 666 & 100 & 4132 & 100 \\
\hline
\end{tabular}

Tabla 6: Tipos de repetición en n1 y \%. Resultados totales por grupos 
El cotejo de estos valores nos da una idea clara de cuáles son los tipos de repetición más habituales en cada grupo. Las únicas divergencias notables que se pueden apreciar conciernen a la repetición simple, a la correferencia y la sustitución, de nuevo, en la prensa down-market. Esta muestra que, aunque la repetición simple es el más importante de sus recursos cohesivos, es un $9 \%$ menos frecuente que la media, siendo otros tipos de repetición, por así decirlo, más sofisticados, como la paráfrasis simple (es decir, el uso de sinónimos) superior a la de los otros dos grupos. Por otra parte, la mayor frecuencia de la correferencia y la sustitución en los diarios de clase down-market hace que las repeticiones léxicas de este grupo sean un $12 \%$ inferiores a las de los otros dos tipos, que son casi idénticas.

A la vista de los resultados estadísticos respecto a vínculos, conexiones y sus tipos podría decirse que todos los grupos de diario muestran las mismas características generales: todos ellos poseen la misma densidad léxica y, por lo tanto, idéntica posibilidad de establecer conexiones, además de una igual relación entre vínculos y conexiones, así como de los tipos generales de estos últimos, predominando claramente las repeticiones léxicas simples. Curiosamente, si hubiera, a pesar de ello, que distinguir las publicaciones entre sí, habida cuenta de las diferencias mencionadas, en lugar de los tres grupos que señala su diferente audiencia o mercado habría, de acuerdo con el análisis de la cohesión léxica, dos tipos de diarios. Por una parte tendríamos los up- y los mid-market, con unos resultados casi idénticos en todos los aspectos mencionados y, por otra, los down-market, cuya peculiaridad reside en poseer un menor número de repeticiones, aunque más relevantes desde el punto de vista de la vinculación, y un mayor porcentaje de conexiones correferenciales o de sustitución, desmintiendo así similitudes debidas al formato tabloide. Resultaría, además, falso el lugar común de que los diarios down-market poseen un estilo más simple que los dirigidos a lectores de clases sociales más altas, ya que estos últimos recurren en la mayoría de las ocasiones $(49,3 \%)$ a utilizar repeticiones de la misma palabra, un supuesto indicativo de mal estilo que Bell (1991a) señala como una de las correcciones más comunes de los editores, mientras que los down-market, aunque también utilizan la repetición simple, la combinan con un alto porcentaje conexiones que evitan la iteración del mismo elemento.

\section{COMPARACIÓN DE LA ORGANIZACIÓN TEXTUAL}

En el caso concreto de los artículos prensa, el estudio de las conexiones léxicas entre las oraciones no sólo sirve para mostrar su organización temática, como Hoey predice, sino que parece también relacionarse con las categorías esquemáticas de la noticia hasta tal punto que las oraciones centrales corresponden con las categorias más relevantes de la misma (Díaz Galán 2002). Por tanto, otra de las características genéricas que el estudio de la cohesión léxica en estos textos nos ha permitido descubrir es la presencia de una organización común a todos los grupos equiparable a la descrita por van Dijk.

Según se refleja en los valores relativos y absolutos que se muestran en las tablas 7 y 8, el análisis de la organización léxica de los textos nos depararía como resultado, para todos los diarios, un artículo prototipo compuesto, al menos, por cuatro oraciones centra- 
les fuertemente vinculadas correspondientes al título y la entradilla, así como al evento principal y a su contexto. Con frecuencia, sería posible también encontrar una quinta oración central expresando los hechos previos, mientras que las consecuencias o reacciones a los mismos, ya sea en forma de hechos previos o de reacciones verbales, serían poco comunes $\mathrm{y}$, finalmente, los comentarios bastante raros:

\begin{tabular}{|c|c|c|c|c|c|c|c|c|c|}
\hline & & & \multicolumn{2}{|c|}{$\begin{array}{c}\text { Up- } \\
\text { Market }\end{array}$} & \multicolumn{2}{|c|}{$\begin{array}{c}\text { Mid- } \\
\text { Market }\end{array}$} & \multicolumn{2}{|c|}{$\begin{array}{l}\text { Down- } \\
\text { Market }\end{array}$} & \multirow[t]{2}{*}{ Total } \\
\hline & & & $\mathrm{N}^{\circ}$ & $\%$ & $\mathrm{~N}^{\circ}$ & $\%$ & $\mathrm{~N}^{\circ}$ & $\%$ & \\
\hline \multicolumn{2}{|c|}{ ESQUEMA } & & 10 & 100 & 10 & 100 & 10 & 100 & 30 \\
\hline \multicolumn{2}{|l|}{ Antetítulo } & & 1 & 10 & - & - & - & - & 1 \\
\hline \multicolumn{2}{|l|}{ Título } & & 9 & 90 & 8 & 80 & 10 & 100 & 27 \\
\hline \multicolumn{3}{|l|}{ Subtítulo } & - & - & 1 & 10 & 1 & 10 & 2 \\
\hline \multicolumn{3}{|l|}{ Entradilla } & 9 & 90 & 9 & 90 & 9 & 90 & 27 \\
\hline \multicolumn{3}{|l|}{ Evento Principal } & 10 & 100 & 10 & 100 & 10 & 100 & 30 \\
\hline \multirow[t]{3}{*}{ Antecedentes } & Circunstancias & Contexto & 7 & 70 & 8 & 80 & 7 & 70 & 22 \\
\hline & & $\begin{array}{l}\text { Hechos } \\
\text { Previos }\end{array}$ & 5 & 50 & 5 & 50 & 7 & 70 & 17 \\
\hline & Historia & & - & - & - & - & - & - & - \\
\hline \multirow{2}{*}{$\begin{array}{l}\text { Consecuencias/ } \\
\text { Reacciones }\end{array}$} & Hechos & & 3 & 30 & 3 & 30 & 5 & 50 & 11 \\
\hline & $\begin{array}{l}\text { Reacciones } \\
\text { Verbales }\end{array}$ & & 6 & 60 & 4 & 40 & - & - & 10 \\
\hline \multicolumn{3}{|l|}{ Expectativas } & - & - & 1 & 10 & 1 & 10 & 2 \\
\hline \multicolumn{3}{|l|}{ Evaluación } & - & - & - & - & - & - & - \\
\hline
\end{tabular}

Tabla 7: Correlación categorías de la noticia / grupos de diarios 


\begin{tabular}{|c|c|c|c|c|c|c|c|}
\hline & & & & \multicolumn{2}{|c|}{$\begin{array}{c}\text { Frecuencia en } \\
\text { todos los } \\
\text { grupos }\end{array}$} & \multicolumn{2}{|c|}{ Oraciones } \\
\hline & & & & $\mathrm{n} 1$ & $\%$ & central & $\begin{array}{c}\text { la más } \\
\text { vinculada }\end{array}$ \\
\hline \multicolumn{4}{|c|}{ ESQUEMA } & 30 & 100 & $\%$ & $\%$ \\
\hline \multirow{5}{*}{ 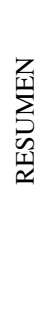 } & Antetítulo & & & 1 & 3 & 100 & - \\
\hline & Título & & & 27 & 90 & 81 & 3 \\
\hline & Subtítulo & & & 2 & 7 & 50 & 3 \\
\hline & Entradilla & & & 27 & 90 & 100 & 45 \\
\hline & Evento Principa & & & 30 & 100 & 100 & 35 \\
\hline \multirow{5}{*}{ 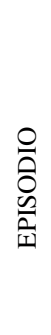 } & \multirow{3}{*}{ Antecedentes } & & Contexto & 22 & 73 & 86 & 1 \\
\hline & & Circunstancias & $\begin{array}{l}\text { Hechos } \\
\text { Previos }\end{array}$ & 17 & 57 & 87 & 8 \\
\hline & & Historia & & - & - & - & - \\
\hline & Consecuencias/ & Hechos & & 11 & 37 & 81 & - \\
\hline & Reacciones & Reacciones Ver & les & 10 & 33 & 60 & 3 \\
\hline \multirow{2}{*}{ 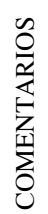 } & Expectativas & & & 2 & 7 & 50 & - \\
\hline & Evaluación & & & - & - & - & - \\
\hline
\end{tabular}

Tabla 8: Correlación categorías de la noticia / oraciones más vinculadas

En la tabla 8, donde se muestra específicamente la correlación entre el esquema de las noticias y las oraciones con más conexiones de todos los textos, podemos comprobar cómo la entradilla y el evento principal, además de ser centrales en la totalidad de los casos, concentran un setenta y cinco por ciento de las oraciones más vinculadas del texto. Es decir, que la oración con mayor número de vínculos y, por tanto, la más relevante de cada uno de ellos corresponderá, en la mayoría de las ocasiones, a una de estas dos categorías (nótese, sin embargo, que la entradilla tiene una ligera preferencia). Estos resultados pueden variar en los casos específicos y así, por ejemplo, la entradilla puede especificar el evento principal (texto 1c), o bien, ésta puede no aparecer (texto 3c) o constar de dos oraciones, en lugar de una (texto 6c). Estos hechos, permitidos, por la flexibilidad en análisis del esquema de la noticia que hicimos notar con anterioridad, no desmienten, sin 
embargo, la posibilidad de predecir, en la mayoría de las noticias, cualquiera que sea el perfil de sus lectores, la relación entre la oración más vinculada y la entradilla o el evento principal.

\section{CONCLUSIÓN}

En este artículo hemos intentado caracterizar genéricamente las noticias publicadas en la prensa británica por medio de sus relaciones cohesivas léxicas. El género, mediador entre el lenguaje y el contexto cultural, se establece, además de atendiendo a la finalidad común de sus ejemplares, a la aparición de otros rasgos, entre los que hemos destacado el uso del léxico y la existencia de un patrón organizativo predeterminado. Tradicionalmente, no obstante, se ha considerado que las noticias varían en aspectos tales como su estilo o su lenguaje debido a la disparidad de la audiencia a la que se dirigen. De acuerdo con nuestro análisis, sin embargo, el hecho de publicarse en diarios de diferentes tipos no es óbice para que estos textos puedan ser considerados como pertenecientes al mismo género informativo.

Los resultados del contraste de dos parámetros, el número y tipos de repeticiones y conexiones, por una parte, y, por otra, la comparación de las categorías de la noticia con la organización léxica del texto en los tres grupos de diarios, nos ha deparado unas diferencias no demasiado significativas entre ellos, respondiendo, así, a unas características que consideramos genéricas por derivarse de la misma intención informativa. A pesar de ello, si hubiera de destacarse alguno, sería sin duda el down-market, ya que posee ciertas peculiaridades, que, aun cuando no lo desvían de la norma general, lo distinguen especialmente del mid-market, el cual, siendo igualmente tabloide, tiene muchas similitudes con el upmarket. Significaría esto que nuestros resultados estan en línea con el estudio de Jucker (1992), y confirmaría que las noticias, indiferentes al grupo de su diario, comparten características genéricas comunes relacionadas con sus conexiones léxicas, aunque mantienen otras específicas, que, en nuestro caso, sólo han afectado a la prensa down-market.

\section{REFERENCIAS BIBLIOGRÁFICAS}

Bell, A. 1991a. The Language of News Media. Oxford: Blackwell.

1991b. "Audience accommodation in the mass media." Contexts of Accommodation. Eds. H. Giles, J. Coupland y N. Coupland. Cambridge: Cambridge University Press, 69-102.

BIBER, D. 1988. Variation across Speech and Writing. Cambridge: Cambridge University Press.

Crystal, D. y D. Davy. 1969. Investigating English Style. Londres: Longman.

DíAZ Galán, A. 2002. "La cohesión léxica como indicador de las categorías esquemáticas de la noticia." RCEI, 44: 245-262

2004. Estudio de la cohesión léxica en las noticias de la prensa londinense. La Laguna: Servicio de Publicaciones de la Universidad de La Laguna. 
Downing, A. 1998. "Organizational frameworks of coherence in discourse and text." Patterns in Discourse and Text. Ensayos del discurso en lengua inglesa. Eds. A. Downing, A. J. Moya Guijarro y J. I. Albentosa Hernández. Cuenca: Ediciones de la Universidad de Castilla-La Mancha. 13-30.

EGgINs, S. 1994. An Introduction to Systemic Functional Linguistics. Londres: Pinter Publishers.

Fowler, J. 1991. Language in the News. Londres: Routledge.

Galtung, J. y M. Ruge. 1973. "Structuring and selecting news." The Manufacture of News: Deviance, Social Problems and the Mass Media. Eds. S. Cohen y J. Young. Londres: Constable. 62-73.

Gutwinsky, W. 1976. Cohesion in Literary Texts. A Study of Some Grammatical and Lexical Features of English Discourse. The Hague: Mouton.

Halliday, M.A.K. 1994. An Introduction to Functional Grammar. Londres: Edward Arnold.

Halliday, M.A.K. y R. Hasan. 1976. Cohesion in English. Hong Kong: Longman.

Hasan, R. 1989. Language, Context and Text: aspects of language in a social-semiotic perspective. Part B. Oxford: Oxford University Press.

Henry, H. 1983. “Are the national newspapers polarizing?” Admap 19.10: 484-491.

. 1987. "National newspaper circulation and their market segmentation". Admap 23.11: 2-24.

Hoey, M. 1991. Patterns of Lexis in Text. Oxford: Oxford University Press.

JuCKer, A. 1992. Social Stylistics. Syntactic Variation in British Newspapers. Nueva York: Mouton de Gruyter.

Kress, G. y T. van LeEuwen. 1998. "Front Pages (The Critical) Analysis of Newspaper Layout." Approaches to Media Discourse. Eds. A. Bell y P. Garret. Oxford: Blackwell, 186-219.

Martin, J. 1992. English Text. System and Structure. Amsterdam: John Benjamins.

Paltridge, B. 1997. Genre, Frames and Writing in Research Settings. Amsterdam: John Benjamins.

Phillips, M. 1989. The Lexical Structure of Text. Discourse Analysis Monographs No 12. Birmingham: English Language Research, University of Birmingham.

Swales, J. 1990. Genre Analysis. Cambridge: Cambridge University Press.

WINTER, E. 1979. "Replacement as a fundamental function of the sentence in context." Forum Linguisticum 4/2: 95-133.

VAN Disk, T. 1977. Text and Context. Londres: Longman. 77-105.

1983. "Estructuras textuales de las noticias de la prensa." Analisi 7/8: 
1987. "Elite discourse and racism." Approaches to Discourse, Poetics and Psychiatry. Eds. I. Zabala, T. A. Van Dijk y M. Díaz-Diocaretz. Amsterdam: Benjamins. 81-122.

1988. News Analysis. Case Studies of International and National News in the Press. Hillsdale: Lawrence Erlbaum.

- 1990. La noticia como discurso. Barcelona: Paidós. Traducción española de News as Discourse (1988. Hillsdale: Lawrence Erlbaum) por Guillermo GAL. 